\title{
Pulsating fronts in periodically modulated neural field models
}

\author{
S. Coombes ${ }^{1, *}$ and C. R. Laing ${ }^{2}$ \\ ${ }^{1}$ School of Mathematical Sciences, University of Nottingham, Nottingham NG7 2RD, United Kingdom \\ ${ }^{2}$ Institute of Information and Mathematical Sciences, Massey University (Albany), Private Bag 102-904, North Shore Mail Centre, Auckland, \\ New Zealand
}

(Received 1 September 2010; revised manuscript received 5 November 2010; published 21 January 2011)

\begin{abstract}
We consider a coarse-grained neural field model for synaptic activity in spatially extended cortical tissue that possesses an underlying periodicity in its microstructure. The model is written as an integrodifferential equation with periodic modulation of a translationally invariant spatial kernel. This modulation can have a strong effect on wave propagation through the tissue, including the creation of pulsating fronts with widely varying speeds and wave-propagation failure. Here we develop a new analysis for the study of such phenomena, using two complementary techniques. The first uses linearized information from the leading edge of a traveling periodic wave to obtain wave speed estimates for pulsating fronts, and the second develops an interface description for waves in the full nonlinear model. For weak modulation and a Heaviside firing rate function the interface dynamics can be analyzed exactly and gives predictions that are in excellent agreement with direct numerical simulations. Importantly, the interface dynamics description improves on the standard homogenization calculation, which is restricted to modulation that is both fast and weak.
\end{abstract}

DOI: 10.1103/PhysRevE.83.011912

PACS number(s): 87.18.Sn, 87.19.1j, 87.19.lq

\section{INTRODUCTION}

The propagation of waves of neural activity across the surface of the brain is known to subserve both natural and pathological neurobiological phenomena. An example of the former is spreading excitation associated with sensory processing [1], while waves in epilepsy are a classic example of the latter [2]. There is now a long history of using integrodifferential neural field models to understand the properties of such waves; for a review of this area we refer the reader to Ref. [3]. For mathematical convenience such models are often assumed to be spatially translationally invariant, and the issue of treating heterogeneity in brain dynamics is avoided. This approach allows one to bring to bear powerful techniques from the study of other translationally invariant physical and biological systems, typically modeled with partial differential equations (PDEs). A review of the analysis of traveling fronts in such local models is given in Ref. [4]. However, it is hard even at a first approximation to view the brain as a homogeneous system, and so there is a pressing need to develop a set of mathematical tools for the study of waves in heterogeneous media that can be used in brain modeling. One common tool used in the PDE community for understanding systems with rapidly oscillating coefficients is homogenization. A particularly nice application of this technique is given in the paper by Keener [5], who studied wave propagation in models of cardiac dynamics with rapidly varying spatial structure. This multiscale approach leads to the study of smoothed or homogenized systems that are averaged over some well-identified microscale. Bressloff [6] was the first to extend this technique to neural field models and to show how to describe fronts that travel through a neural model with a periodically modulated microstructure. However, it is important to emphasize that as a perturbation technique, homogenization theory requires that modulation on the micro scale be both low in amplitude and rapidly varying in

*stephen.coombes@nottingham.ac.uk space. Interestingly, there are other techniques in the applied mathematical sciences that have been developed for the study of waves in random media that might also translate well to heterogeneous neural field models. In particular, asymptotic techniques, stochastic homogenization, variational methods, and large deviation methods, as reviewed by Xin [7,8], are all worthy of consideration. Of all these techniques, the one developed by Shigesada $e t$ al. [9] for treating periodic waves in a heterogeneous (periodically modulated) single-species population model of reaction-diffusion type seems the most appealing. This method uses linearized information from the leading edge of a traveling periodic wave to obtain wave speed estimates, generalizing the approach commonly used for the study of homogeneous PDEs [10]. One of the results we present here is the generalization of this technique to cover nonlocal interactions and its subsequent application to neural field models. Another result is the development of a new technique for studying the evolution of interfaces in heterogeneous neural fields: a technique that may translate back to the PDE community.

In Sec. II we introduce the mathematical model of a one-dimensional neural field with a periodically modulated nonlocal interaction kernel. Numerical simulation is used to illustrate the possibility of a traveling pulsating front solution in an excitatory model with a sinusoidally modulated exponentially decaying connectivity. Next, in Sec. III we use linear analysis to develop a minimum wave speed calculation of traveling pulsating fronts, for a specific piecewise-linear firing rate function. This analysis is related to mathematical approaches for studying Kolmogorov-Petrovskii-Piscuinovtype equations $[11,12]$ but is able to cope with the nonlocality of neural field models. Even though the calculation uses information from only the leading edge of a wave, it gives wave speed predictions in very good agreement with those from direct numerical simulations. In Sec. IV we consider a Heaviside firing rate function and focus on time-independent spatially varying solutions and show how to determine existence and stability of pinned front solutions. A fully nonlinear 
analysis of traveling fronts is developed in Sec. V based on tracking the evolution of level set contours of the neural activity. For a Heaviside firing rate this interface dynamics simplifies considerably and the dynamics for a traveling front is determined by a single scalar nonlinear ordinary differential equation (ODE). For weak modulation this ODE can be analyzed using standard perturbation arguments and the wave speed of a pulsating front is easily calculated. Once again, the theory is in excellent agreement with numerical simulations and, in contrast to results obtained using homogenization theory, can describe wave behavior even when the period modulation of the spatial kernel is not rapid. Finally, in Sec. VI we summarize and discuss possible extensions of this work.

\section{THE MODEL}

Many current coarse-grained models of neural tissue trace their origins back to seminal work in the 1970s by Wilson and Cowan $[13,14]$ and Amari $[15,16]$. In one spatial dimension the simplest single population model that describes the evolution of neural activity $u=u(x, t)$, with $x \in \mathbb{R}$ and $t \in \mathbb{R}^{+}$, is

$$
u_{t}=-u+\psi, \quad \psi(x, t)=\int_{-\infty}^{\infty} \mathrm{d} y W(x, y) f \circ u(y, t) .
$$

The function $f$ represents the firing rate of the tissue and is often chosen to have a sigmoidal form. The weight kernel $W(x, y)$ represents anatomical connectivity between point $x$ and point $y$ in the tissue, and the presence of this function means that the model has a nonlocal structure. For a more comprehensive discussion of such models, their generalization, and their use in neuroscience, we refer the reader to Ref. [17]. In this paper we mostly focus on a very specific form of heterogeneity, namely, one where the connectivity has the following product structure:

$$
W(x, y)=W(|x-y|) J(y), \quad J(y)=J(y+\sigma),
$$

where $J$ is some $\sigma$-periodic function. The use of periodically modulated, translationally invariant kernels of this form is particularly relevant to modeling primary visual cortex, which is known to have a periodic microstructure on the millimeter scale, reviewed in some detail in the paper by Bressloff [6]. For later convenience we use a Fourier representation for the real periodic function $J$ and write

$$
J(x)=\sum_{n} J_{n} \mathrm{e}^{2 \pi i n x / \sigma}, \quad J_{-n}=J_{n}^{\dagger},
$$

where $J_{n} \in \mathbb{C}$ and $\dagger$ denotes complex conjugation. To provide a sense of the type of solutions that the model defined by Eqs. (1), (2) and (3) can support, we first present a direct numerical simulation for the choice

$$
W(x)=\frac{1}{2} \mathrm{e}^{-|x|}, \quad J(x)=J_{0}+\epsilon \sin (2 \pi x / \sigma),
$$

and $f(u)=H(u-h)$, where $H$ is the Heaviside function.

Figure 1 shows a space-time plot of $u(x, t)$ and a wave that connects a low-activity state to a high-activity state. Although there is an easily identified front that separates these two states, it does not travel with constant speed and in fact oscillates periodically in a comoving frame (whose speed is chosen in an appropriate fashion, described in Sec. III). In the wake of the front, one also sees a periodic spatial pattern. We call such

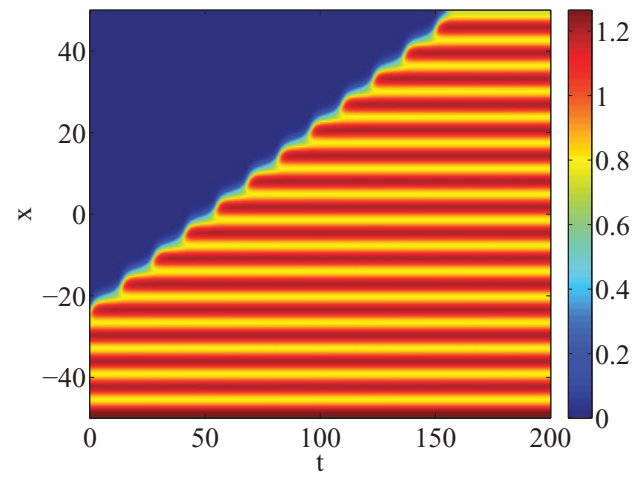

FIG. 1. (Color online) An example of a pulsating front solution of Eqs. (1) and (2) for $W(x)=\mathrm{e}^{-|x|} / 2, J(x)=J_{0}+\epsilon \sin (2 \pi x / \sigma)$, with $f(u)=H(u-h)$, using parameter values $J_{0}=1, \epsilon=0.4, h=0.3$, and $\sigma=2 \pi$.

a wave a pulsating front. Numerical simulations with such a firing rate function show that with increasing $\epsilon$, such fronts can fail to propagate, instead becoming pinned, in qualitative (though not quantitative) agreement with predictions from homogenization theory. We provide an analysis of pinned fronts in Sec. IV, but before that we consider their propagating counterparts, for a specific, piecewise-linear, firing rate function.

\section{TRAVELING PERIODIC WAVE}

We focus on solutions of the type shown in Fig. 1, where enough time has passed so that a periodic traveling wave can develop defined by the conditions $u(x, t)=u(x+\sigma, t+T)$, $\lim _{x \rightarrow \infty} u(x, t)=0$, and $\lim _{x \rightarrow-\infty} u(x, t)=q(x)$, where $q(x)$ is a stationary (spatially periodic) function. Note that the solution repeats itself in time $T$ if it is observed at two points a distance $\sigma$ apart. We define a speed $c$ of the wave according to $c=\sigma / T$. Waves of this type have previously been studied by Shigesada et al. [9] in a PDE model of population dynamics in a heterogeneous environment. These authors developed wave speed estimates after linearizing about the leading edge of the wave. Here we adapt their arguments and develop a method for studying nonlocal systems that can be applied to neural field equations. To apply their methods we work with the piecewise-linear firing rate function given by

$$
f(u)=f_{1}(u) \equiv \begin{cases}0, & \text { if } u<0 \\ \gamma u, & \text { if } 0 \leqslant u \leqslant 1 / \gamma \\ 1, & \text { if } u>1 / \gamma .\end{cases}
$$

Note that for a more general sigmoidal form of firing rate, estimates of the wave speed would rely on information from across the whole solution and not just the leading edge. Later, in Secs. IV and V, we work with a Heaviside firing rate, which is obtained from Eq. (5) in the limit $\gamma \rightarrow \infty$.

\section{A. Dispersion relationship and minimum speed formula}

For an exponentially decaying $W(x)$ and firing rate functions for which $f(0)=0$, the leading edge of a pulsating front solution is given simply by $u=0$. This is often called a pulled front (as it is pulled by the unstable state, $u=0$ ). 
Linearizing around this solution gives the following linear integrodifferential equation:

$$
u_{t}=-u+\psi, \quad \psi(x, t)=\gamma \int_{-\infty}^{\infty} \mathrm{d} y W(x, y) u(y, t),
$$

where $\gamma=\lim _{u \searrow 0} f^{\prime}(u)$. It is natural to look for solutions to Eq. (6) in the form $u(x, t)=w(\xi) v(x)$, with $\xi=x-c t$, where $\lim _{\xi \rightarrow \infty} w(\xi)=0$ and $v(x)=v(x+\sigma)$. Here we recognize $\xi$ as a comoving coordinate (in a frame of unknown speed $c)$, so that $u(x, t)$ has a product structure that separates into a traveling wave part and a spatial part. Substitution of this ansatz into Eq. (6) gives

$$
\begin{aligned}
-c v(x) \frac{\mathrm{d} w}{\mathrm{~d} \xi}= & -v(x) w(\xi) \\
& +\gamma \int_{-\infty}^{\infty} \mathrm{d} y W(|y|) J(x-y) w(\xi-y) v(x-y) .
\end{aligned}
$$

For the choice $w(\xi)=\alpha \mathrm{e}^{\lambda \xi}, \lambda \in(-\infty, 0)$ (so that solutions decay to $u=0$ as $\xi \rightarrow \infty$ ), and writing $v(x)=\sum_{n} v_{n} \mathrm{e}^{2 \pi i n x / \sigma}$, we find

$$
\begin{aligned}
(1-c \lambda) \sum_{n} v_{n} \mathrm{e}^{2 \pi i n x / \sigma}= & \gamma \sum_{m, p} J_{m} v_{p} \mathrm{e}^{2 \pi i x(m+p) / \sigma} \\
& \times \widehat{W}(2 \pi(m+p) / \sigma, \lambda),
\end{aligned}
$$

where

$$
\widehat{W}(k, \lambda)=\int_{-\infty}^{\infty} \mathrm{d} y W(|y|) \mathrm{e}^{-\lambda y} \mathrm{e}^{-i k y} .
$$

Note that $\widehat{W}^{\dagger}(-k, \lambda)=\widehat{W}(k, \lambda)$. Projecting Eq. (8) onto $\mathrm{e}^{-2 \pi i l x / \sigma}$ and integrating over $x$ gives an infinite system of linear equations for the amplitudes $v_{l}$ as

$$
(1-c \lambda) v_{l}=\gamma \widehat{W}(2 \pi l / \sigma, \lambda) \sum_{m} J_{m} v_{l-m} .
$$

Introducing the infinite matrix $\mathcal{W}(c, \lambda)$ with components

$$
[\mathcal{W}(c, \lambda)]_{l m}=\gamma J_{l-m} \widehat{W}(2 \pi l / \sigma, \lambda)-(1-c \lambda) \delta_{l m},
$$

we see that the system defined by (10) has a nontrivial solution if 0 is an eigenvalue of $\mathcal{W}(c, \lambda)$. This matrix represents an unbounded linear operator $\mathcal{W}: l_{2}^{\mathbb{Z}} \rightarrow l_{2}^{\mathbb{Z}}$. Here, $l_{2}^{\mathbb{Z}}$ is the Hilbert space of complex sequences $\left(\ldots, z_{-1}, z_{0}, z_{1}, \ldots\right)$ with $\sum_{k \in \mathbb{Z}}\left|z_{k}\right|^{2}<\infty$. The spectrum of $\mathcal{W}$ consists of a countable number of eigenvalues, all of which are of finite multiplicity. The number 0 is an eigenvalue of $\mathcal{W}(c, \lambda)$ if and only if $\operatorname{ker} \mathcal{W} \neq\{0\}$, where 0 denotes the null vector in $l_{2}^{\mathbb{Z}}$. In order to carry out practical calculations we truncate the system of equations (10) and consider only indices $l$ from $-N, \ldots, N$. This reduces the infinite eigenvalue problem of operator $\mathcal{W}$ to the calculation of a finite determinant. This truncation technique has a sound mathematical foundation and has often been used to study Hill's infinite matrix, especially as it arises in the analysis of the Mathieu equation (see, e.g., Ref. [18]) or its time-delayed extension [19]. (In practice, we used $N=20$ and checked that our results were insensitive to increases in $N$.)
Following Ref. [11], we now introduce a minimum propagation speed $c^{*}$ as

$$
c^{*}=\inf \{c \mid \operatorname{det} A(c, \lambda)=0, \lambda<0\},
$$

where $A(c, \lambda)$ is interpreted as the truncated (finite) version of Eq. (11). From this we can also define a critical decay rate $\lambda^{*}$ via $\operatorname{det} A\left(c^{*}, \lambda^{*}\right)=0$. If we consider a traveling wave that arises from initial data with $\lim _{x \rightarrow \infty} u(x, 0)=\alpha \mathrm{e}^{\lambda x}$, with $\lambda<0$, then around $u=0$ we expect solutions of the form

$$
u(x, t)=\alpha_{1} \mathrm{e}^{\lambda^{*}\left(x-c^{*} t\right)}+\alpha_{2} \mathrm{e}^{\lambda(x-c(\lambda) t)},
$$

where $c(\lambda)$ is defined by $\operatorname{det} A(c, \lambda)=0$. If the initial data decay sufficiently fast, namely, $\lambda<\lambda^{*}$, then the first term in Eq. (13) dominates and we expect waves to emerge with the minimum wave speed $c^{*}[20,21]$. If the initial data have a slow decay, then the resulting wave will have a minimum speed determined by $c(\lambda)$.

Consider, for example, the choice (4), so that $J_{1}=\epsilon /(2 i)$ and

$$
\widehat{W}(k, \lambda)=\frac{1}{(1+\lambda+i k)(1-\lambda-i k)}, \quad|\lambda|<1 .
$$

In this case the (finite) matrix $A$ is tridiagonal, with entries

$$
\begin{aligned}
A_{m, m} & =\gamma \widehat{W}(2 \pi m / \sigma, \lambda) J_{0}-1+c \lambda, \\
A_{m, m-1} & =\gamma \widehat{W}(2 \pi m / \sigma, \lambda) J_{1}=-A_{m, m+1},
\end{aligned}
$$

and the fixed point at $u=0$ is unstable for small $\epsilon$ when $-1+$ $\gamma J_{0}>0$ [as can be seen by considering spatially coherent perturbations of the form $u(x, t)=\beta \exp (\lambda t)$ in Eq. (6) and demanding that $\operatorname{Re} \lambda>0$ ].

\section{B. Numerical results}

A plot of $c$ as a function of $\lambda$, defined by the relation $\operatorname{det} A(c, \lambda)=0$, is shown in Fig. 2 for various values of $\epsilon$. Here it can be seen that $c=c(\lambda)$ has a well-defined minimum $c^{*}$, which increases with increasing $\epsilon$. In Fig. 3 we trace $c^{*}$ as a function of $\epsilon$ for different values of $\gamma$ and make a comparison

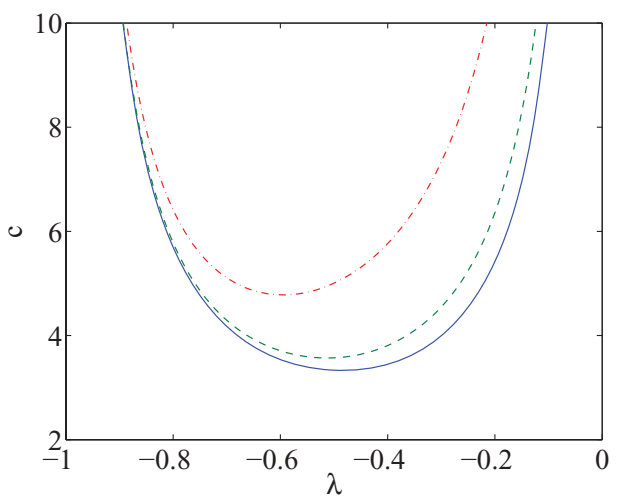

FIG. 2. (Color online) A plot of the wave speed estimate $c$ as a function of $\lambda$ defined by $\operatorname{det} A(c, \lambda)=0$ with $N=20$ for three values of $\epsilon . \epsilon=1.5$ [dash-dotted (red) curve], $\epsilon=0.5$ [dashed (green) curve], and $\epsilon=0$ [solid (blue) curve]. Other parameters are $\gamma=2$, $J_{0}=1$, and $\sigma=2 \pi$. 


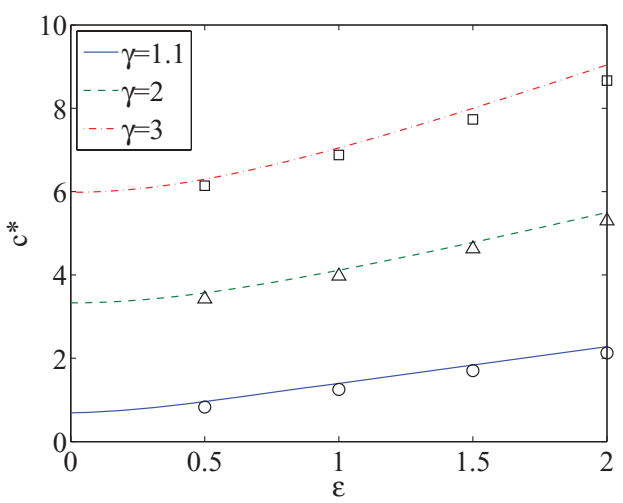

FIG. 3. (Color online) Minimum wave speed $c^{*}$ as a function of $\epsilon$ for three values of $\gamma$. Other parameter values are $J_{0}=1$ and $\sigma=2 \pi$. Also shown are values measured from numerical simulations for $\gamma=1.1$ (circles), $\gamma=2$ (triangles), and $\gamma=3$ (squares).

of the wave speed estimate with direct numerical simulations. For small values of $\epsilon$ these agree very well, and even for $\epsilon \sim O(1)$ we still find good agreement.

In Fig. 4 we show some corresponding plots for $c^{*}$ as a function of $\sigma$ with various values of $\epsilon$, to emphasize that good agreement between analysis and simulations holds over a large window of parameter space. In the next two sections we move away from the use of linearized information to estimate wave speeds and consider both stationary and moving patterns, showing how to analyze wave speeds in the full nonlinear model when the firing rate is a Heaviside function.

\section{TIME-INDEPENDENT SOLUTIONS}

A stationary (time-independent) solution of Eq. (1) satisfies the equation

$$
q(x)=\int_{-\infty}^{\infty} \mathrm{d} y W(x, y) f[q(y)] .
$$

The stability of this solution can be determined by considering perturbations to $q(x)$ of the form $u(x) \mathrm{e}^{\lambda t}$. In this case

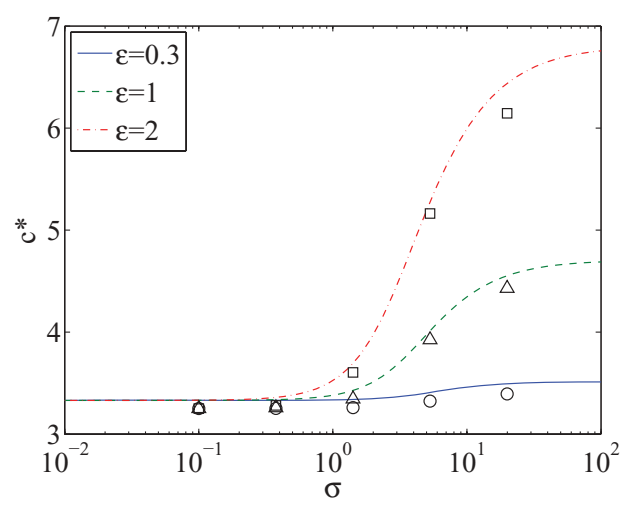

FIG. 4. (Color online) Minimum wave speed $c^{*}$ as a function of $\sigma$ ( $\log$ scale) for three values of $\epsilon$. Other parameter values are $J_{0}=1$ and $\gamma=2$. Also shown are values measured from numerical simulations for $\epsilon=2$ (squares), $\epsilon=1$ (triangles), and $\epsilon=0.3$ (circles). linearizing around $q(x)$ gives the equation

$$
(1+\lambda) u(x)=\int_{-\infty}^{\infty} \mathrm{d} y W(x, y) f^{\prime}[q(y)] u(y) .
$$

For bounded solutions $u(x)$ of Eq. (17) we say that $q(x)$ is stable if $\operatorname{Re} \lambda<0$.

Since pinned pulsating front solutions provide a connection between a periodic spatial pattern (in the wake of the front) and a steady state (ahead of the front), it is natural to first consider the construction of a pure spatially periodic solution. Since solutions of Eq. (16) are rarely available in closed form for an arbitrary firing rate (although see Ref. [22] for a recent discussion of how to analyze a particular class of sigmoids), we focus on the special case of a Heaviside for which solutions are easily constructed.

\section{A. Periodic solutions}

For a Heaviside firing rate

$$
f(u)=f_{2}(u) \equiv H(u-h)
$$

$\sigma$-periodic solutions with $q(x)>h$ for all $x$ are given simply by

$$
q(x)=\int_{-\infty}^{\infty} \mathrm{d} y W(x, y) .
$$

For example, with the choice Eq. (4), then

$$
q(x)=J_{0}+\frac{\epsilon}{1+(2 \pi / \sigma)^{2}} \sin (2 \pi x / \sigma) .
$$

To ensure that $q(x)>h$ we must have that $J_{0}-|\epsilon| /[1+$ $\left.(2 \pi / \sigma)^{2}\right]>h$. For this problem the right-hand side of Eq. (17) vanishes (since the solution never crosses $h$ ) and we have simply that $\lambda=-1$ and the solution is stable.

\section{B. Pinned front}

For a Heaviside firing rate function $f(u)=f_{2}(u)$, connections between periodic solutions, described in Sec. IV A, and the homogeneous state $q(x)=0$ can easily be constructed, and describe pinned fronts. An example is shown in Fig. 5. If $q(x)>h$ for $x<\eta$, where $0 \leqslant \eta<\sigma$ and $q(x) \leqslant h$

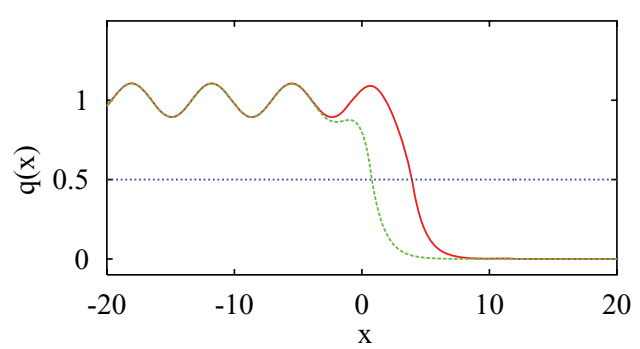

FIG. 5. (Color online) An example of a pair of pinned fronts in a model with $W(x)=\mathrm{e}^{-|x|} / 2, J(x)=J_{0}+\epsilon \sin (2 \pi x / \sigma)$, and $f(u)=$ $H(u-h)$. The solid (dashed) line shows a stable (unstable) solution. Here $J_{0}=1, \epsilon=0.3, \sigma=2 \pi$, and $h=0.5$. 
otherwise, we have simply that

$$
q(x)=\int_{-\infty}^{\eta} \mathrm{d} y W(x, y) .
$$

Imposing the condition $q(\eta)=h$ fixes the parameter conditions for the existence of such a solution. For example, with the choice Eq. (4), then

$$
q(x)= \begin{cases}q_{+}(x), & x \geqslant \eta, \\ q_{-}(x), & x<\eta,\end{cases}
$$

where

$$
\begin{gathered}
q_{+}(x)=h \mathrm{e}^{\eta-x}, \\
q_{-}(x)=h+\frac{J_{0}}{2}\left(1-\mathrm{e}^{-(\eta-x)}\right)+\frac{\epsilon}{2 \sqrt{1+(2 \pi / \sigma)^{2}}} \\
\times\left[\sin (2 \pi x / \sigma+\phi)-\mathrm{e}^{-(\eta-x)} \sin (2 \pi \eta / \sigma+\phi)\right],
\end{gathered}
$$

and $\tan \phi=2 \pi / \sigma$ subject to the constraint

$$
2 h=J_{0}+\frac{\epsilon}{\sqrt{1+(2 \pi / \sigma)^{2}}} \sin (2 \pi \eta / \sigma-\phi) .
$$

As a function of $\eta$, Eq. (25) has either two solutions or none. In the latter case traveling fronts arise, invading the region where $u=0$ if $h$ is too low and retreating if $h$ is too high. A plot of $q(\eta)$ and $q=h=$ const. is shown in Fig. 6 to illustrate the possible solutions of $q(\eta)=h$ (where the line and curve meet). To ensure $q(x)>h$ for $x<0$, we must have that $J_{0}>|\epsilon| / \sqrt{1+(2 \pi / \sigma)^{2}}$. To determine stability we note that $f_{2}^{\prime}[q(y)]=\delta(q(y)-h)=\delta(y-\eta) /\left|q^{\prime}(\eta)\right|$ and Eq. (17) reduces to

$$
(1+\lambda) u(x)=W(x, \eta) \frac{u(\eta)}{\left|q^{\prime}(\eta)\right|} .
$$

Demanding nontrivial solutions $[u(\eta) \neq 0]$, we must have that

$$
\lambda=-1+\frac{W(\eta, \eta)}{\left|q^{\prime}(\eta)\right|}=-1+\frac{W(0) J(\eta)}{|W(0) J(\eta)-L(\eta)|},
$$

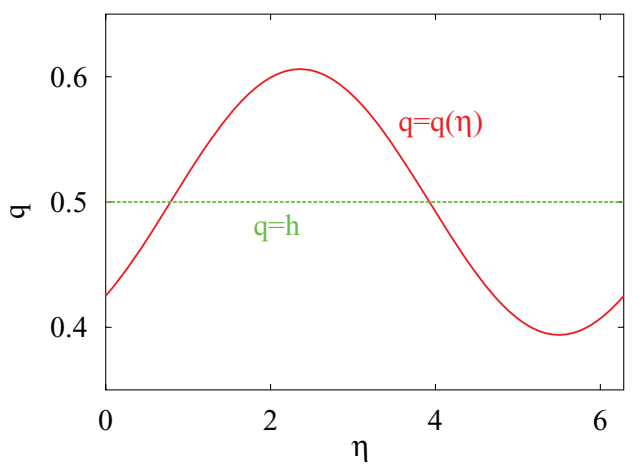

FIG. 6. (Color online) A plot of the existence condition on the firing threshold $h$ for solutions shown in Fig. 5. Linear stability analysis shows that solutions, corresponding to the points at which $q(\eta)=h$, with $\mathrm{d} q(\eta) / \mathrm{d} \eta<0$, are stable and those with $\mathrm{d} q(\eta) / \mathrm{d} \eta>0$ are unstable. where

$$
L(\eta)=\int_{0}^{\infty} \mathrm{d} y W(y) J^{\prime}(\eta-y)=\frac{\mathrm{d} q(\eta)}{\mathrm{d} \eta} .
$$

Hence, solutions with $\mathrm{d} q(\eta) / \mathrm{d} \eta<0$ are stable and those with $\mathrm{d} q(\eta) / \mathrm{d} \eta>0$ are unstable. For the example in Fig. 5 we see from Fig. 6 that, of the two possible solutions, it is the one with the largest $\eta$ that is stable.

\section{INTERFACE DYNAMICS}

Motivated by the form of the pulsating front in Fig. 1 we seek to describe the properties of this solution solely in terms of the behavior at the front edge that separates high activity from low. If the front is not pulsating (which is the case in the absence of period modulation of the connectivity), then in a traveling wave frame (of the same speed as the wave) the rising edge of the front may be identified with a single (traveling wave) coordinate. For a pulsating front this point is no longer stationary in time and instead oscillates. We now show how to derive the dynamics for this interface between high- and low-activity states.

In a comoving frame, model (1) takes the form $u=u(\xi, t)$, where $\xi=x-c_{0} t$ for some fixed $c_{0}$, and

$$
-c_{0} u_{\xi}+u_{t}=-u+\psi
$$

where

$$
\psi(\xi, t)=\int_{-\infty}^{\infty} \mathrm{d} y W\left(\xi+c_{0} t, y\right) f \circ u\left(y-c_{0} t, t\right) .
$$

We define a moving interface (level set) according to

$$
u\left[\xi_{0}(t), t\right]=h
$$

for some constant $h$. Here we are assuming that there is only one point on the interface (though, in principle, we could consider a set of points). Differentiation of Eq. (31) gives an exact expression for the velocity of the interface in the form

$$
\dot{\xi}_{0}=-\left.\frac{u_{t}}{u_{\xi}}\right|_{\xi=\xi_{0}(t)} .
$$

Focusing now on the case of a Heaviside firing rate with $f(u)=f_{2}(u)$ means that for a pulsating front solution with $u>h$ for $\xi<\xi_{0}$ Eq. (30) takes the simple form

$$
\psi(\xi, t)=\int_{-\infty}^{\xi_{0}+c_{0} t} \mathrm{~d} y W\left(\xi+c_{0} t, y\right) .
$$

\section{A. Perturbation analysis}

Consider a perturbation around the unmodulated case and write $W(x, y)=W(|x-y|)[1+\epsilon K(y)][K(y)=K(y+\sigma)]$ for some small parameter $\epsilon$. For $\epsilon=0$ there is a traveling front $q(\xi)$ given by the solution of

$$
-c_{0} \frac{\mathrm{d} q}{\mathrm{~d} \xi}=-q+\psi, \quad \psi(\xi)=\int_{\xi}^{\infty} \mathrm{d} y W(|y|),
$$

where the speed $c_{0}$ is determined by $q(0)=h$. For small $\epsilon$ we assume that the slope of the traveling front varies sufficiently slowly so that we may make the convenient approximation 
$\left.u_{\xi}\right|_{\xi=\xi_{0}(t)}=\left.q_{\xi}\right|_{\xi=0}$. In this case we have, using Eqs. (29) and (34), that

$$
\begin{gathered}
\left.u_{t}\right|_{\xi=\xi_{0}(t)}=\int_{-\infty}^{\xi_{0}+c_{0} t} \mathrm{~d} y W\left(\xi_{0}+c_{0} t, y\right)-\int_{0}^{\infty} \mathrm{d} y W(|y|), \\
\left.u_{\xi}\right|_{\xi=\xi_{0}(t)}=\frac{1}{c_{0}}\left(h-\int_{0}^{\infty} \mathrm{d} y W(|y|)\right)
\end{gathered}
$$

Substitution of Eqs. (35) and (36) into Eq. (32) gives

$$
\dot{\xi}_{0}=\epsilon c_{0} \frac{\int_{0}^{\infty} \mathrm{d} y W(|y|) K\left(\xi_{0}+c_{0} t-y\right)}{h-\int_{0}^{\infty} \mathrm{d} y W(|y|)} .
$$

For the choice $K(x)=\sin (2 \pi x / \sigma)$ and $W(x)=\mathrm{e}^{-|x|} / 2$, the time-dependent speed of the front is then given by $c_{0}[1+$ $\left.\epsilon a\left(\xi_{0}, t\right)\right]$, where $c_{0}=(1-2 h) /(2 h)$ and

$$
a\left(\xi_{0}, t\right)=A \sin \left[\frac{2 \pi}{\sigma}\left(\xi_{0}+c_{0} t\right)-\phi\right],
$$

with

$$
A=\frac{1}{2 h-1} \frac{1}{\sqrt{1+(2 \pi / \sigma)^{2}}}, \quad \tan \phi=\frac{2 \pi}{\sigma} .
$$

Pulsating fronts are $T$-periodic solutions of the nonautonomous ordinary differential equation, (37), with $\xi_{0}(t)=$ $\xi_{0}(t+T)$. Introducing $x_{0}=\xi_{0}+c_{0} t$ with $x_{0} \in[0, \sigma]$, we may solve for the trajectory using

$$
\int_{0}^{x_{0}} \frac{\mathrm{d} x}{1+\epsilon A \sin (2 \pi x / \sigma-\phi)}=c_{0} t .
$$

Using a half-angle substitution we may evaluate this to give

$$
c_{0} t=\left.\frac{\sigma}{\pi} \frac{1}{\alpha} \tan ^{-1} \frac{z}{\alpha}\right|_{z_{0}(0)+\epsilon A} ^{z_{0}(t)+\epsilon A}, \quad \alpha^{2}=1-\epsilon^{2} A^{2},
$$

where $z_{0}(t)=\tan \left[\left(2 \pi x_{0}(t) / \sigma-\phi\right) / 2\right]$ and $x_{0}(0)=0$. A periodic pulsating front with speed $c=\sigma / T$ can be found by demanding that $\sigma=x_{0}(T)$. Substitution of this condition into Eq. (41) shows that the speed of the pulsating front is given by

$$
c=c_{0} \sqrt{1-\epsilon^{2} A^{2}} \text {. }
$$

Hence, a propagating wave is only supported if $|\epsilon|<1 /|A|$.

\section{B. Numerical results}

A plot of the wave speed as a function of the spatial scale $\sigma$ is shown in Fig. 7. We see excellent agreement between the theoretical predictions from the interface dynamics approach and direct numerical simulations. Although our analysis in this case is restricted to small $\epsilon$, we have not had to make any assumptions about the scale of periodic modulation as determined by the parameter $\sigma$. In contrast, a homogenization analysis would require both small $\epsilon$ and a periodic modulation that occurs on a smaller length scale than the correlation length of $W(x)$ (which is set to unity here). Following the calculation by Bressloff in Ref. [6], a naive application of homogenization theory gives

$$
c=c_{0} \sqrt{1-\epsilon^{2} B^{2}}, \quad B=\frac{1}{2 h-1} \frac{\sigma}{2 \pi} .
$$

As expected, the result from the interface approach, Eq. (42), recovers that from homogenization theory, given by Eq. (43),

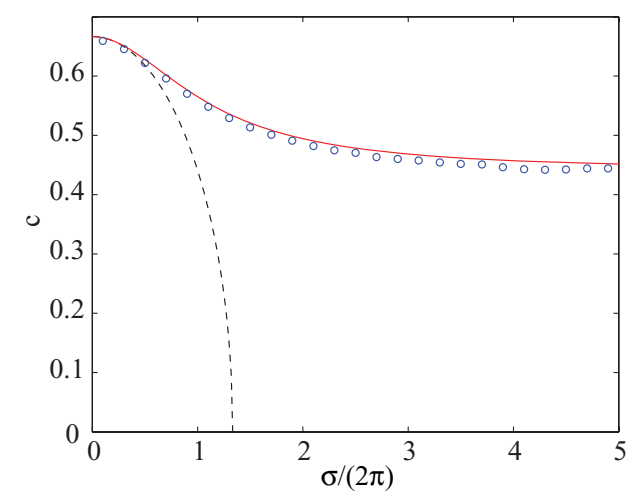

FIG. 7. (Color online) Solid (red) line: result from an interface dynamics analysis given by Eq. (42). Circles show results measured from simulations. Dashed (black) line: result from homogenization theory given by Eq. (43). Parameters are $\epsilon=0.3$ and $h=0.3$.

in the limit $\sigma \rightarrow 0$. However, as illustrated in Fig. 7 the homogenization result gives poor results for $\sigma$ away from 0 . This has already been pointed out by Schmidt et al. [23], who use different arguments to derive Eq. (42). In their perturbation analysis they assume that the front is approximately homogeneous in a very small time window. Several other applications of the ideas in this section are possible, and we explore these briefly here.

\section{Other forms of spatial modulation}

Thus far we have only considered spatial modulation of the coupling function $W$. Here we consider several other possible forms of spatial modulation. Suppose we replace Eq. (1) with

$$
\begin{aligned}
& u_{t}(x, t)=-u(x, t)[1+\epsilon K(x)]+\psi(x, t), \\
& \psi(x, t)=\int_{-\infty}^{\infty} \mathrm{d} y W(|x-y|) f_{2} \circ u(y, t) .
\end{aligned}
$$

where $K$ is periodic. Performing the same analysis as in Sec. V A, one obtains the equation

$$
\dot{\xi}_{0}=\epsilon c_{0} \frac{h K\left(\xi_{0}+c_{0} t\right)}{h-\int_{0}^{\infty} \mathrm{d} y W(|y|)},
$$

and using the same functions $W$ and $K$ as in that section results in the expression

$$
c=c_{0} \sqrt{1-\epsilon^{2} A_{1}^{2}},
$$

where $A_{1}=2 h /(2 h-1)$. Note that in this expression $c$ is independent of $\sigma$, the spatial wavelength of the modulation. Comparison of Eq. (47) with results from numerical simulations of Eqs. (44) and (45) are shown in Fig. 8. We see good agreement for small $\epsilon$, as expected.

Another possible way of including spatial modulation is to study the system

$$
\begin{gathered}
\frac{u_{t}(x, t)}{1+\epsilon K(x)}=-u(x, t)+\psi(x, t), \\
\psi(x, t)=\int_{-\infty}^{\infty} \mathrm{d} y W(|x-y|) f_{2} \circ u(y, t),
\end{gathered}
$$




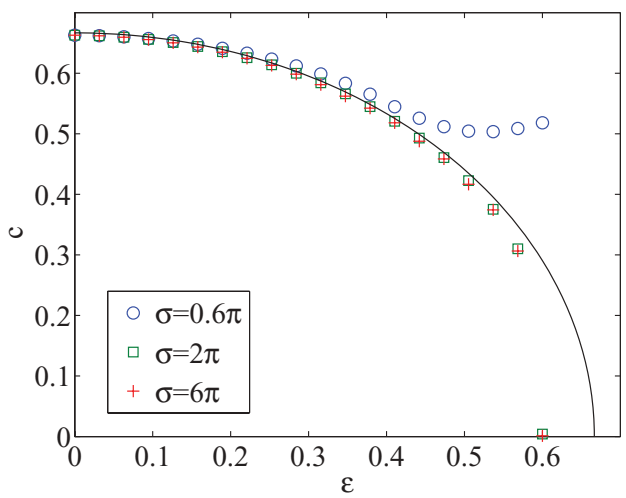

FIG. 8. (Color online) Solid (black) line: result from an interface dynamics analysis given by Eq. (47). Symbols show the results of numerical simulations for different values of $\sigma$. Parameters are $h=0.3$.

in which the time scale for the dynamics is modulated. Performing an analysis similar to the foregoing, one obtains

$$
c=c_{0} \sqrt{1-\epsilon^{2}}
$$

In this case, the critical value of $\epsilon$ beyond which waves are predicted not to travel is $\epsilon=1$, independent of both the spatial wavelength of modulation and the value of $h$, the threshold in the firing rate function. Figure 9 shows a comparison of Eq. (50) with results from numerical simulations of Eqs. (48) and (49). The agreement is very good, although it appears to become worse as $\sigma \rightarrow 0$.

A third alternative is the system

$$
\begin{gathered}
u_{t}=-u+\psi+\epsilon K(x), \\
\psi(x, t)=\int_{-\infty}^{\infty} \mathrm{d} y W(|x-y|) f_{2} \circ u(y, t),
\end{gathered}
$$

modeling a spatially dependent stimulus, as originally proposed by Amari [16]. For functions $K$ and $W$ as before, we have

$$
c=c_{0} \sqrt{1-\epsilon^{2} A_{2}^{2}},
$$

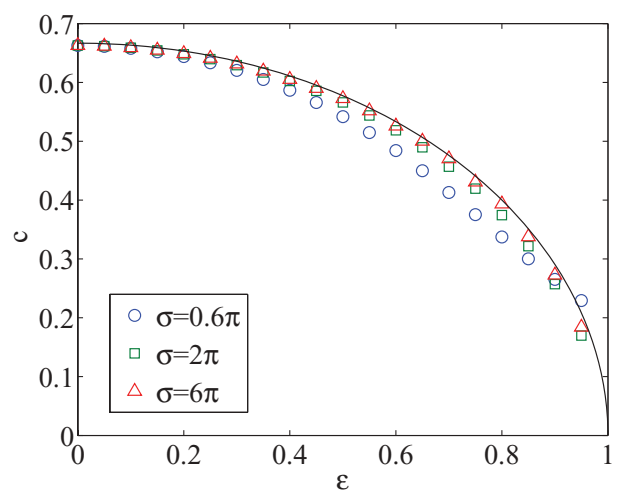

FIG. 9. (Color online) Solid (black) line: result from an interface dynamics analysis given by Eq. (50). Symbols show the results of numerical simulations for different values of $\sigma$. Parameters are $h=0.3$.

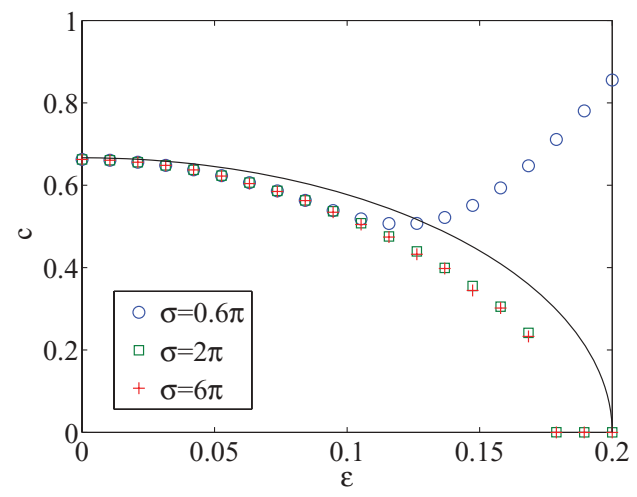

FIG. 10. (Color online) Solid (black) line: result from an interface dynamics analysis given by Eq. (53). Symbols show the results of numerical simulations for different values of $\sigma$. Parameters are $h=0.3$.

where $A_{2}=2 /(2 h-1)$. Note that in this expression $c$ is independent of $\sigma$, the spatial wavelength of the modulation. Figure 10 shows a comparison of Eq. (53) with results from numerical simulations of Eqs. (51) and (52).

\section{DISCUSSION}

In this paper we have shown that the biologically interesting case of a periodically modulated neural field model is amenable to mathematical analysis using two complementary techniques. One of these techniques uses linearization to obtain wave speed estimates for a particular piecewise-linear firing rate function and is a generalization of approaches used in the PDE community. The other is a novel interface dynamics, especially relevant to neural field models where the firing rate switches rapidly between low and high values about some threshold. Although we have focused on describing front propagation, both techniques carry over to the study of traveling pulses. The linearization approach cannot distinguish between the speed of a front and the speed of a pulse, while the interface approach provides more information, as it tracks both the leading edge and the trailing back of the pulse (via a pair of ODEs). For a recent study of propagating pulses in inhomogeneous neural media using averaging and homogenization theory, we refer the reader to Kilpatrick et al. [24].

The two firing rate functions we considered are the piecewise-linear $f_{1}(u)$ [Eq. (5)] and the shifted Heaviside $f_{2}(u)$ [Eq. (18)]. Both are approximations to the type of biophysically more plausible sigmoidal function that is often used $[13,25]$. To obtain the results in Sec. III on traveling fronts, we needed $f(0)=0$ and $\gamma \equiv \lim _{u \searrow 0} f^{\prime}(u)>1$, thus $f=f_{1}$ was an appropriate choice. Since the value of $\gamma$ is the only information about $f(u)$ for $u$ positive that is used in the analysis in Sec. III, the results there will equally apply to other firing rate functions, such as

$$
f(u)=f_{3}(u) \equiv \begin{cases}0, & \text { if } u<0, \\ \tanh (\gamma u), & \text { if } 0 \leqslant u,\end{cases}
$$

[for which $\left.\lim _{u \rightarrow \infty} f(u)=1\right]$ or

$$
f(u)=f_{4}(u) \equiv \begin{cases}0, & \text { if } \quad u<0, \\ \gamma \tanh u, & \text { if } 0 \leqslant u,\end{cases}
$$




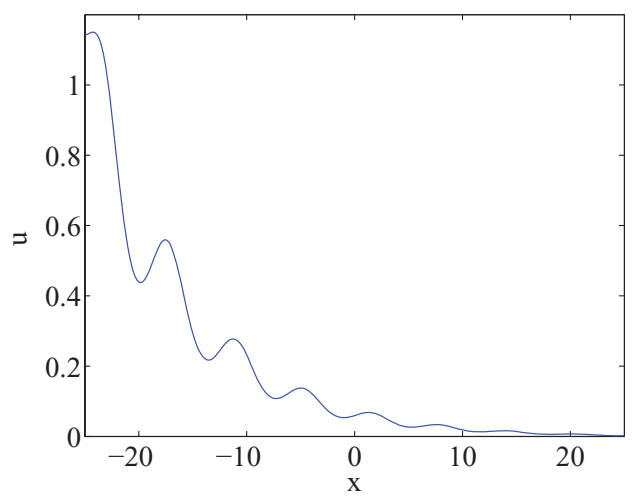

FIG. 11. (Color online) A stationary solution of Eqs. (1) and (2) for $f=f_{1}$. Parameters are $\gamma=0.95, J_{0}=1, \sigma=2 \pi$, and $\epsilon=0.3$.

[for which $\lim _{u \rightarrow \infty} f(u)=\gamma$ ], both of which have $\lim _{u \searrow 0} f^{\prime}(u)=\gamma$. Numerical simulations using $f(u)=f_{3}(u)$ or $f_{4}(u)$ give results very similar to those shown in Figs. 3 and 4 , confirming this (results not shown). Note also that nontrivial stationary solutions are possible for the choice $f=f_{1}$ and $\gamma<1$; see Fig. 11 for an example.

In contrast, the choice of $f=f_{2}$ made the calculations in Secs. IV and V straightforward. For example, $f[q(y)]$ in Eq. (16) is either 0 or 1 , and $f^{\prime}[q(y)]$ in Eq. (17) is proportional to a Dirac $\Delta$ function at the point at which $q(y)=h$. Comparing Figs. 4 and 7 it is interesting to note that the speed of a front increases as $\sigma$ is increased when $f=f_{1}$ and decreases as $\sigma$ is increased when $f=f_{2}$. This demonstrates that the qualitative behavior of a model such as Eqs. (1) and (2) can depend on the exact form of the nonlinear function $f$. The only reasonable way to compare results obtained for $f=f_{1}$ and $f=f_{2}$ would be to take $\gamma \rightarrow \infty$ in $f_{1}$ and $h \rightarrow 0$ in $f_{2}$. However, the result would be $f(u)=H(u)$, the unshifted Heaviside, for which the speed of a front in the unmodulated case is infinite $\left[c_{0}=(1-2 h) /(2 h)\right]$. Thus, a meaningful comparison is not really possible, and our methods and results can be regarded as complementary to one another. In general, of course, one would like to derive results for an arbitrary sigmoidal function $f$.

The shifted Heaviside function $f_{2}$ is more appropriate for use in studying spreading patterns in two spatial dimensions, where interfaces will be more complicated than simple points. For example, in the case of a spreading spot the interface would be a closed curve. Importantly, the formalism of the level set approach that we have used in one spatial dimension carries over and the evolution of interfaces can be solely prescribed in terms of a dynamics on the interface (at least when the firing rate is a Heaviside). Indeed an interface description is a very natural way to treat neural field dynamics with other forms of heterogeneity, such as patchy connections [25-28].

It is worth emphasizing that fronts or interfaces in periodic media are deterministic problems. In contrast to this, there are many instances in neuroscience where lack of data means that it is more natural to model the inhomogeneous environment as a random process. It is thus an open challenge to understand responses of the corresponding stochastic tissue model, though ideas developed for studying PDE models with random parameters may be an appropriate starting point [8]. One recent advance in the analysis of random traveling waves in the Nagumo equation with multiplicative noise has been made by Lord and Thümmler [29], and it would be interesting to explore the extension of their work to neural field models.
[1] G. B. Ermentrout and D. Kleinfeld, Neuron 29, 33 (2001).

[2] B. W. Connors and Y. Amitai, in Epilepsy: Models, Mechanisms and Concepts, edited by P. A. Schwartzkroin (Cambridge University Press, Cambridge, 1993), pp. 388-424.

[3] S. Coombes, Biol. Cybern. 93, 91 (2005).

[4] W. van Saarloos, Phys. Rep. 386, 29 (2003).

[5] J. Keener, Physica D 136, 1 (2000).

[6] P. C. Bressloff, Physica D 155, 83 (2001).

[7] J. Xin, SIAM Rev. 42, 161 (2000).

[8] J. Xin, An Introduction to Fronts in Random Media, Surveys and Tutorials in the Applied Mathematical Sciences (Springer, New York, 2009).

[9] N. Shigesada, K. Kawasaki, and E. Teramoto, Theoret. Popul. Biol. 30, 143 (1986).

[10] O. Diekmann, J. Math. Biol. 6, 109 (1978).

[11] W. Hudson and B. Zinner, in Boundary Value Problems for Functional-Differential Equations, edited by J. Henderson (World Scientific, Singapore, 1995), pp. 187-199.

[12] H. Berestycki and F. Hamel, Commun. Pure Appl. Math. 55, 949 (2002).

[13] H. R. Wilson and J. D. Cowan, Biophys. J. 12, 1 (1972).

[14] H. R. Wilson and J. D. Cowan, Kybernetik 13, 55 (1973).
[15] S. Amari, Biol. Cybern. 17, 211 (1975).

[16] S. Amari, Biol. Cybern. 27, 77 (1977).

[17] S. Coombes, NeuroImage 52, 731 (2010).

[18] D. W. Jordan and P. Smith, Nonlinear Ordinary Diferential Equations, 3rd ed. (Oxford University Press, New York, 1999).

[19] T. Insperger and G. Stepan, J. Dyn. Syst. Meas. Control 125, 166 (2003).

[20] K. P. Hadeler and F. Rothe, J. Math. Biol. 2, 251 (1975).

[21] F. Rothe, Rocky Mt. J. Math. 11, 617 (1981).

[22] S. Coombes and H. Schmidt, Discrete Cont. Dyn. Syst. Ser. A 28, 1369 (2010).

[23] H. Schmidt, A. Hutt, and L. Schimansky-Geier, Physica D 238, 1101 (2009).

[24] Z. Kilpatrick, S. E. Folias, and P. C. Bressloff, SIAM J. Appl. Dyn. Syst. 7, 161 (2008).

[25] S. Coombes, N. A. Venkov, L. Shiau, I. Bojak, D. T. J. Liley, and C. R. Laing, Phys. Rev. E 76, 051901 (2007).

[26] V. K. Jirsa and J. A. S. Kelso, Phys. Rev. E 62, 8462 (2000).

[27] P. C. Bressloff, Physica D 185, 131 (2003).

[28] C. A. Brackley and M. S. Turner, Biol. Cybern. 100, 371 (2009).

[29] G. J. Lord and V. Thümmler, e-print arXiv:1006.0428v1 (2010). 